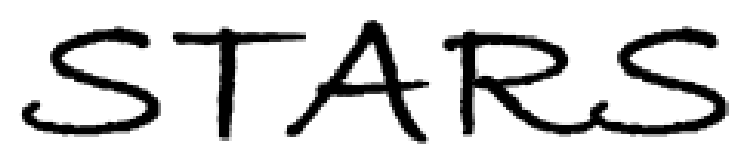

University of Central Florida

STARS

Faculty Bibliography 2000s

Faculty Bibliography

$1-1-2005$

\title{
Wave dynamics in optically modulated waveguide arrays
}

Mark J. Ablowitz

Keith Julien

Ziad H. Musslimani

University of Central Florida

Michael I. Weinstein

Find similar works at: https://stars.library.ucf.edu/facultybib2000

University of Central Florida Libraries http://library.ucf.edu

This Article is brought to you for free and open access by the Faculty Bibliography at STARS. It has been accepted for inclusion in Faculty Bibliography 2000s by an authorized administrator of STARS. For more information, please contactSTARS@ucf.edu.

\section{Recommended Citation}

Ablowitz, Mark J.; Julien, Keith; Musslimani, Ziad H.; and Weinstein, Michael I., "Wave dynamics in optically modulated waveguide arrays" (2005). Faculty Bibliography 2000s. 4936.

https://stars.library.ucf.edu/facultybib2000/4936

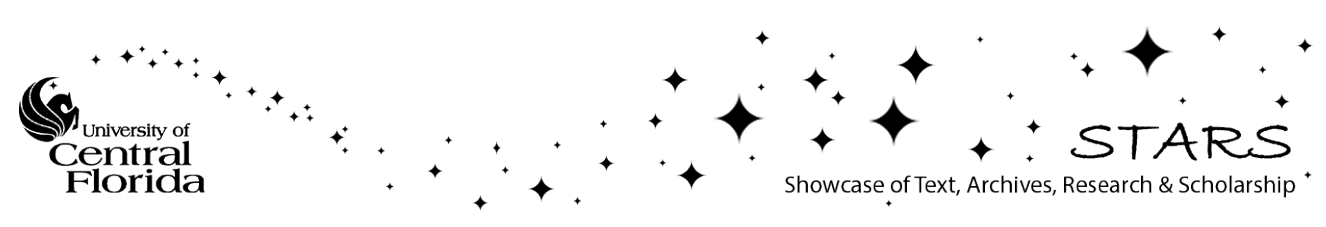




\title{
Wave dynamics in optically modulated waveguide arrays
}

\author{
Mark J. Ablowitz, ${ }^{1}$ Keith Julien, ${ }^{1}$ Ziad H. Musslimani, ${ }^{2}$ and Michael I. Weinstein ${ }^{3}$ \\ ${ }^{1}$ Department of Applied Mathematics, University of Colorado, Campus Box 526, Boulder, Colorado 80309-0526, USA \\ ${ }^{2}$ Department of Mathematics, University of Central Florida, Orlando, Florida 32816, USA \\ ${ }^{3}$ Department of Applied Physics and Applied Mathematics, Columbia University, 200 S.W. Mudd - MC4701 New York, \\ New York 10027, USA
}

(Received 20 January 2005; published 11 May 2005)

\begin{abstract}
A model describing wave propagation in optically modulated waveguide arrays is proposed. In the weakly guided regime, a two-dimensional semidiscrete nonlinear Schrödinger equation with the addition of a bulk diffraction term and an external "optical trap" is derived from first principles, i.e., Maxwell equations. When the nonlinearity is of the defocusing type, a family of unstaggered localized modes are numerically constructed. It is shown that the equation with an induced potential is well-posed and gives rise to localized dynamically stable nonlinear modes. The derived model is of the Gross-Pitaevskii type, a nonlinear Schrödinger equation with a linear optical potential, which also models Bose-Einstein condensates in a magnetic trap.
\end{abstract}

DOI: 10.1103/PhysRevE.71.055602

PACS number(s): 42.65.Wi

Wave propagation in nonlinear periodic structures displays unique phenomena that are absent in homogeneous media. The interplay between periodicity and nonlinearity can lead to the formation of discrete or lattice solitons, which were predicted theoretically in the context of optical waveguide arrays [1] and then experimentally observed in [2]. Until recently, discrete solitons were considered experimentally in one-dimensional geometry [2]. However, by making use of the photorefractive screening nonlinearity one can "write" either one- or higher-dimensional optical waveguide arrays by interfering pairs of plane waves [3]. Indeed, such localized structures were experimentally observed in twodimensional geometries [4].

In this paper we study wave propagation in optically modulated waveguide arrays, starting from the full timeharmonic three-dimensional Maxwell's equations. For the case where the periodic modulation along the $y$ direction is much larger than the periodic modulation along the $x$ direction we derive, using multiscale asymptotic analysis, a semidiscrete nonlinear Schrödinger equation with the addition of bulk diffraction term and an external "optical trap." When the nonlinearity is of the defocusing type (where in the absence of modulation no finite energy solitons are known) unstaggered localized modes are numerically constructed. The fundamental properties such as the well-posedness of the equation, existence, and the dynamical stability associated with a special class of localized wave solutions, i.e., stationary wave, or ground state, are discussed. The semidiscrete model is derived from the scalar nonlinear Helmholtz equation. Below we briefly outline the justification for neglecting vectorial effects under certain physical assumptions. A more general and detailed study of scalar and vector semidiscrete nonlinear Schrödinger (NLS) type models will be given elsewhere.

We begin by considering the three-dimensional Maxwell equations governing time-harmonic solutions of frequency $\omega_{0}$

$$
\nabla^{2} \mathbf{E}-\boldsymbol{\nabla}(\boldsymbol{\nabla} \cdot \mathbf{E})+k_{0}^{2}(\mathbf{E}+\mathbf{P})=0, \quad k_{0}=\frac{\omega_{0}}{c} .
$$

Here, $\boldsymbol{\nabla}=\partial_{x} \hat{\mathbf{i}}+\partial_{y} \hat{\mathbf{j}}+\partial_{z} \hat{\mathbf{k}}, \mathbf{E}=\mathbf{E}\left(\mathbf{x} ; \omega_{\mathbf{0}}\right)$ denotes the complex envelope of the electric field, $\mathbf{P}=\mathbf{P}\left(\mathbf{E}(\mathbf{x}) ; \omega_{0}\right)$ denotes the polarization field, containing both linear and nonlinear responses; we further assume the nonlinear polarization to be of Kerr type [5] where the second component of the polarization is given by

$$
P_{2}=\chi E_{2}+\delta\left(\left(\left|E_{1}\right|^{2}+(1+\gamma)\left|E_{2}\right|^{2}+\left|E_{3}\right|^{2}\right) E_{2}+\gamma\left(E_{1}^{2}+E_{3}^{2}\right) E_{2}^{*}\right)
$$

where $\gamma$ is a constant, $\delta$ is proportional to the nonlinear index change of refraction, and $\chi$ is a function of $x$ and $y$; the other polarization components are found by cyclically changing the indices $(1 \rightarrow 2 \rightarrow 3 \rightarrow 1)$. We consider propagation in the $z$ direction through a photonic structure (invariant in $z$ ) having nontrivial spatial variations in the $(x, y)$ plane due to $\chi$. A schematic of the kind of transverse structure we consider is given in Fig. 1. This structure has a rapid periodic variation in $x$ and a slow modulation in $y$. In nondimensional terms, this corresponds to the assumed form $\chi=\chi\left(x, \varepsilon^{1 / 2} y\right)$, where $\varepsilon$ is a small dimensionless parameter. The period in $x$ is of order 1 whereas a typical distance in $y$ is of order $\varepsilon^{-1 / 2}$. We further assume that the nondimensional nonlinear index of refraction is small in size $[O(\varepsilon)]$. Then, analysis of Maxwell's equations (1) shows that $E_{3} / E_{2}=O(\varepsilon)$ and $E_{1} / E_{2}=O\left(\varepsilon^{3 / 2}\right)$ and to leading order

$$
\partial_{z} E_{3}=-\partial_{y} E_{2} \text {. }
$$

Then the second component of Maxwell's equations (1) leads to the following nonlinear Helmholtz equation

$$
\nabla^{2} \Psi+f^{2}(x, y) \Psi+\varepsilon \eta|\Psi|^{2} \Psi=0
$$

where $\Psi$ is the envelope wave function, which is proportional to the optical field $E_{2}\left(\nabla^{2}=\partial_{x}^{2}+\partial_{y}^{2}+\partial_{z}^{2}\right) \cdot f^{2}(x, y)=1+\chi$ is the linear refractive index of the waveguide structure, $\eta$ is proportional to $\delta$ and $\operatorname{sgn} \eta=+1$ and $\operatorname{sgn} \eta=-1$ correspond to, respectively, the cases of self-focusing and self-defocusing 


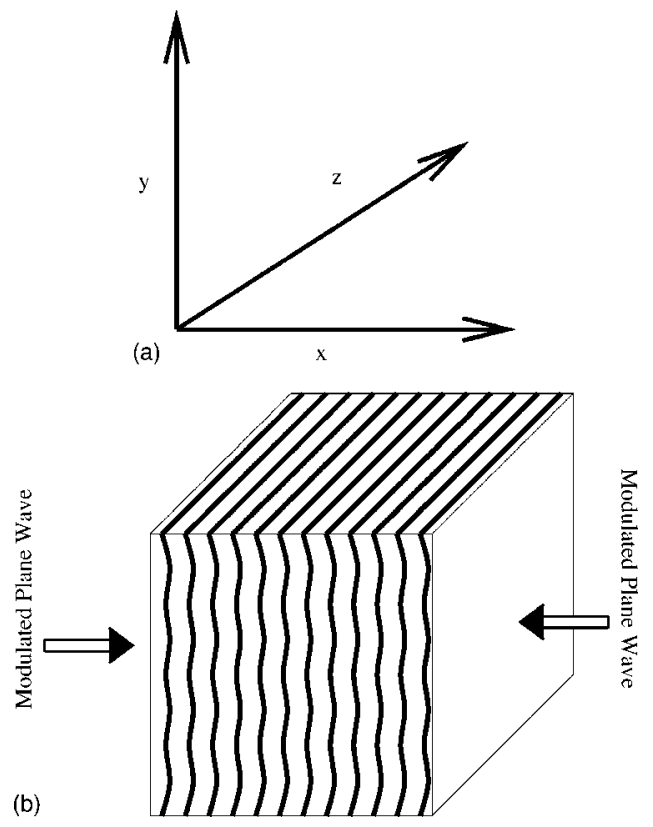

FIG. 1. A typical modulated waveguide array.

nonlinearity. We assume that the linear refractive index appearing in (4) takes the form

$$
f^{2}(x, y)=\mathcal{F}^{2}(x)+\varepsilon a \mathcal{F}_{p}^{2}\left(x, \varepsilon^{1 / 2} y\right),
$$

where $a= \pm 1 . \mathcal{F}^{2}(x)$ is the refractive index of a grating structure in the $x$ longitudinal coordinate, which may be viewed as a superposition of spatial translates of a basic waveguide with index profile $F_{0}^{2}(x)$, which we assume to be single moded. Thus, $\mathcal{F}^{2}(x) \sim \sum_{m} F_{m}^{2}(x), F_{m}(x)=F_{0}(x-m D)$. Here, $\varepsilon \mathcal{F}_{p}^{2}\left(x, \varepsilon^{1 / 2} y\right)$ is a weak modulation of refractive index (slow in $y$ and fast in $x$ ). One can create a photonic structure of this type by illuminating a photorefractive crystal with a pair of interfering one-dimensional plane waves weakly modulated along the $y$ direction with a wavelength larger than the wavelength along the $x$ direction. We now analyze wave propagation in a nonlinear optical two-dimensional array and discuss the physical phenomena that result.

We exploit the weak nonlinearity (small $\varepsilon$ ) in (4) and (5) to construct a multiple scale expansion of the envelope, $\Psi$. We seek $\Psi$ as a superposition of $x$ - translates of the isolated single mode wave function with slowly varying amplitudes [6]

$$
\Psi \approx \sum_{m=-\infty}^{+\infty} A_{m}(Z, Y) \psi_{m}(x) e^{i \mu z},
$$

where $Z=\varepsilon z$ and $Y=\varepsilon^{1 / 2} y$ are slow propagation and modulation scales, respectively. Here, $\psi(x)$ is the single waveguide mode and $\mu$ its corresponding eigenvalue

$$
\frac{d^{2} \psi}{d x^{2}}+\left(F_{0}^{2}(x)-\mu^{2}\right) \psi=0,
$$

and $\psi_{m}(x)=\psi(x-m D)$. Substituting the expansion (6) into the Helmholtz equation (4) and making use of (7), we find that the corrections to (6) are small provided the projections of

$$
\begin{aligned}
& \sum_{m=-\infty}^{+\infty}\left[2 i \varepsilon \mu \psi_{m} \frac{\partial A_{m}}{\partial Z}+\psi_{m}\left(\mathcal{F}^{2}(x)-F_{m}^{2}(x)\right) A_{m}+\varepsilon \psi_{m} \frac{\partial^{2} A_{m}}{\partial Y^{2}}\right. \\
& \left.\quad+a \varepsilon \mathcal{F}_{p}^{2}(x, Y) \psi_{m} A_{m}+\varepsilon \eta \sum_{m^{\prime}, m^{\prime \prime}=-\infty}^{+\infty} \psi_{m} \psi_{m^{\prime}} \psi_{m^{\prime \prime}}^{*} A_{m} A_{m^{\prime}} A_{m^{\prime \prime}}^{*}\right] \\
& \quad=0
\end{aligned}
$$

onto all $\psi_{j}$ are of order $\varepsilon^{\delta}, \delta>1$. This yields (see also [6])

$$
i \frac{\partial A_{n}}{\partial Z}+C\left(A_{n+1}+A_{n-1}\right)+\gamma \frac{\partial^{2} A_{n}}{\partial Y^{2}}+a V_{n}(Y) A_{n}+\kappa\left|A_{n}\right|^{2} A_{n}=0,
$$

where $\quad \varepsilon C=1 / c_{0} \int\left(\mathcal{F}^{2}(x)-F_{n \pm 1}^{2}(x)\right) \psi_{n \pm 1} \psi_{n}^{*} d x, \quad V_{n}(Y)$ $=1 / c_{0} \int \mathcal{F}_{p}^{2}(x, Y)\left|\psi_{n}\right|^{2} d x, \quad \kappa=\eta / c_{0} \int\left|\psi_{n}\right|^{4} d x, \quad$ and $\quad c_{0}$ $=2 \mu \int\left|\psi_{n}\right|^{2} d x ; \gamma=1 /(2 \mu)$. Note that we are considering the regime where only nearest neighbor waveguides contribute to order $\varepsilon$. Equation (9) governs the slow evolution of $A_{n}$ in a weakly modulated optically induced waveguide array. Next we examine linear propagation and then highlight some physical nonlinear phenomena that are predicted by the model (9). For the ideal one-dimensional waveguide array $\left[\gamma=\kappa=V_{n}(Y) \equiv 0\right]$, the propagating field experiences discrete diffraction due to optical tunneling to adjacent sites and exhibits a typical discrete diffraction pattern with the intensity mainly concentrated in the outer lobes [7]. However, in the presence of modulation $\left(\gamma \neq 0\right.$ and $\left.V_{n} \neq 0\right)$, and in the quasitwo-dimensional configuration (when modulating along the $y$ direction), the waveguide action prevents the beam from diffracting. It should also be noted that a similar derivation in the case when two fields are initially present, i.e., nontrivial $E_{1}, E_{2}$ leads to a vector system whose first component satisfies,

$$
\begin{aligned}
& i \frac{\partial A_{n}^{(1)}}{\partial Z}+C\left(A_{n+1}^{(1)}+A_{n-1}^{(1)}\right)+\gamma \frac{\partial^{2} A_{n}^{(1)}}{\partial Y^{2}}+a V_{n}(Y) A_{n}^{(1)} \\
& \quad+\kappa\left[(1+\gamma)\left|A_{n}^{(1)}\right|^{2}+\left|A_{n}^{(2)}\right|^{2}\right] A_{n}^{(1)}+\gamma A_{n}^{(1)^{2}} A_{n}^{(2) *}=0,
\end{aligned}
$$

and the second equation is obtained by cyclically changing the indices $(1 \rightarrow 2 \rightarrow 1)$. In a future publication we will give the derivation in detail. We now discuss the results obtained for the model (9) which are depicted in Figs. 2-4. First, in both self-focusing and self-defocusing cases, propagating beams of any finite power do not collapse or filament. This is in contrast to the continuum analog, the two-dimensional cubic-focusing NLS equation, whose solutions with sufficient initial power are well known to develop singularities at a finite distance into a bulk Kerr propagation medium [8]. That the semidiscrete character inhibits collapse, (see Fig. 4), can be understood by an argument based on the conserved integrals of (9) 


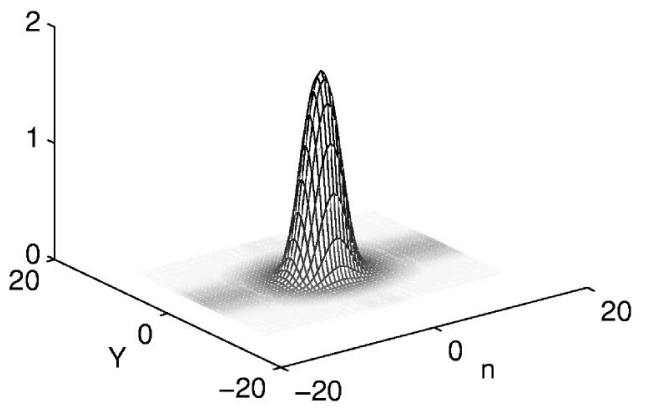

FIG. 2. Localized semidiscrete, two-dimensional soliton solution to Eq. (12) in the presence of a semidiscrete two-dimensional trap $(\alpha=\beta=1 / 2)$ for the defocusing nonlinearity. The parameters are $C=2, \nu=4, \gamma=1$, and $\kappa=-1$.

$$
\begin{gathered}
\mathcal{N}=\sum_{n} \int\left|A_{n}\right|^{2} d Y \\
\mathcal{H}=C \sum_{n} \int\left|A_{n+1}-A_{n}\right|^{2}+\left|\partial_{Y} A_{n}\right|^{2}-a V_{n}\left|A_{n}\right|^{2}-\frac{\kappa}{2}\left|A_{n}\right|^{4} d Y .
\end{gathered}
$$

That no singularity can form as the wave form propagates through increasing $Z$ is a direct consequence of the $Z$ - independence of $\mathcal{N}$ and $\mathcal{H}$ and the semidiscrete SobelevGagliardo-Nirenberg inequality (dSNG) for functions $f_{n}(Y)$ defined on the integer lattice times the real continuum [9-11]: Let $\vec{f}=\left(f_{n}(Y)\right), \quad \vec{f}=\left(f_{n+1}(Y)\right)$ and $\|\vec{f}\|_{p}^{p}=\Sigma_{n} \int\left|f_{n}\right|^{p} d Y$. Then, there is a universal constant $C_{*}>0$ such that for all $\vec{f}$

$$
\|\vec{f}\|_{4}^{4} \leqslant C_{*}\left\|\partial_{Y} \vec{f}\right\|_{2}\|\vec{f}-\tau \vec{f}\|_{2}\|\vec{f}\|_{2}^{2} .
$$

In particular, the $Z$ independence of $\mathcal{N}$ and $\mathcal{H}$ and the inequality (11) together imply upper bounds on $\left\|\partial_{Y} \vec{f}(Z)\right\|_{2}$ and $|\vec{f}(Z, Y)|$ in terms of $\mathcal{H}$ and $\mathcal{N}$ (which are independent of $Z$ and $Y$ ). These bounds break down when passing to the continuum limit. It should be remarked that in the defocusing case, $\gamma \kappa<0$, when $V_{n}(Y) \equiv 0$, there are no localized nonlinear modes. In this case, a finite energy concentration diffractively spreads and attenuates in amplitude. However, the optical trapping potential, $V_{n}(Y)$, introduces the possibility of stable bright solitonlike states. Indeed, the existence and importance of such nonlinear defect modes has been studied in the context of a plasma model [12] and in the trapping of nonlinear pulses in fiber gratings with localized defects [13]. Such nonlinear defect modes are solutions to Eq. (9) of the form $A_{n}=B_{n} e^{-i v Z}$ and satisfy

$$
\nu B_{n}+C\left(B_{n+1}+B_{n-1}\right)+\gamma \frac{\partial^{2} B_{n}}{\partial Y^{2}}-V_{n}(Y) B_{n}+\kappa\left|B_{n}\right|^{2} B_{n}=0,
$$

where $\nu>0$ is the propagation constant. In the above, we took $a=-1$ and $\eta=-1$. The existence and stability of these states follows from their variational characterization as local minima of the energy functional $\mathcal{H}$ subject to fixed total power, $\mathcal{N}$. Here too, the inequality (11) plays a role in that it implies the boundedness from below of the constrained en-

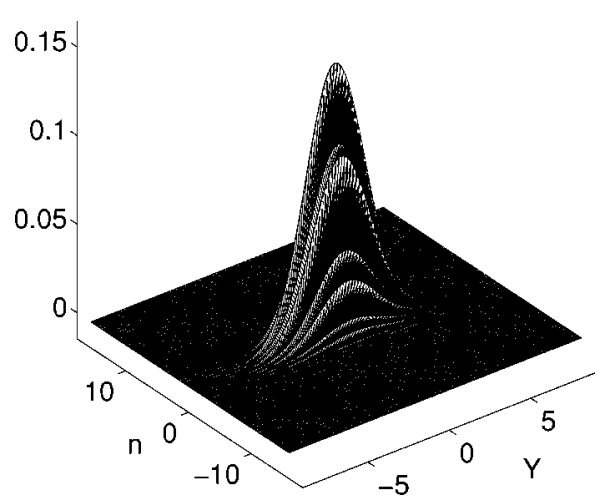

FIG. 3. Localized semidiscrete, two-dimensional soliton solution to Eq. (12) in the presence of a one-dimensional "discrete trap" $(\alpha=1 / 2 ; \beta=0.1)$ for the defocusing nonlinearity. The parameters are $C=2, \nu=4, \gamma=1$, and $\kappa=-1$.

ergy. For simplicity, in our numerics we consider a perturbed index, which is locally parabolic (which can be induced by a weak sinusoidal refractive index $), \mathcal{F}_{p}^{2}(x, Y)=\tilde{\alpha} x^{2}+\widetilde{\beta} Y^{2}$ leading to the induced potential

$$
V_{n}(Y)=\alpha n^{2}+\beta Y^{2}
$$

To numerically construct the bound states solutions to Eq. (12) we first define the Fourier transform $\mathrm{F}$ and its inverse $\mathbb{F}^{-1}$

$$
\begin{gathered}
\hat{B}(k, q)=\mathbb{F}\left[B_{n}(Y)\right]=\sum_{n=-\infty}^{+\infty} \int_{-\infty}^{+\infty} B_{n}(Y) e^{-i(q n+k Y)} d Y, \\
B_{n}(Y)=\mathbb{F}^{-1}[\hat{B}(k, q)]=\frac{1}{(2 \pi)^{2}} \int_{-\infty}^{+\infty} \int_{-\pi}^{+\pi} \hat{B}(k, q) e^{+i(q n+k Y)} d k d q .
\end{gathered}
$$

The idea of the method (see also Ref. [14]) is to make the change of variables: $B_{n}(Y)=\theta Q_{n}(Y), \quad \hat{B}(k, q)=\theta \hat{Q}(k, q)$, where $\theta \neq 0$ is a constant to be determined from a consistency condition. Taking the Fourier transform on Eq. (12) and using the above change of variables we get

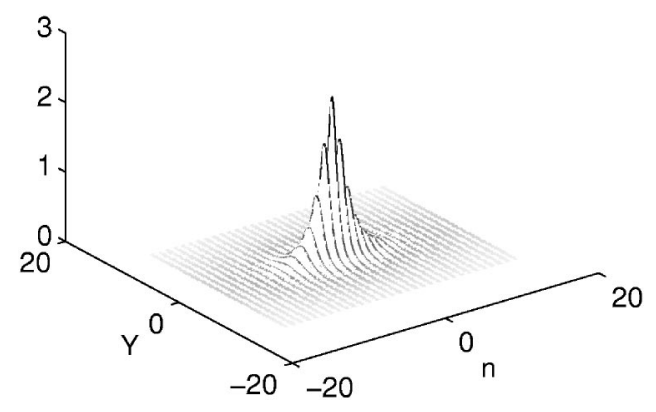

FIG. 4. Localized semidiscrete, two-dimensional soliton solution to Eq. (12) without a trap $\left[V_{n}(Y) \equiv 0\right]$ for the focusing nonlinearity. The parameters are $C=2, \nu=1, \gamma=1$, and $\kappa=+1$. 


$$
\Omega(k, q) \hat{Q}(k, q)-\mathbb{F}\left[V_{n}(Y) Q_{n}(Y)\right]=-\theta^{2} \mathbb{F}\left[\kappa\left|Q_{n}\right|^{2} Q_{n}\right],
$$

where $\Omega(k, q)=\nu+2 C \cos (q)-\gamma k^{2}$. Multiplying Eq. (16) by $\hat{Q}^{*}(k, q)$ and integrating over the $(k, q)$ space, we find

$$
\theta^{2}=\frac{-\int \hat{Q}^{*}(k, q)\left\{\Omega(k, q) \hat{Q}(k, q)-\mathbb{F}\left[V_{n}(Y) Q_{n}(Y)\right]\right\} d k d q}{\int \hat{Q}^{*}(k, q) \mathbb{F}\left[\kappa\left|Q_{n}\right|^{2} Q_{n}\right] d k d q} .
$$

Since $\Omega(k, q)$ vanishes for $\nu=-2 C \cos (q)+\gamma k^{2}$, we add and subtract $(r+2 C) \hat{Q}(k, q)$ in Eq. (16) where $r$ is an arbitrary positive number. Then the iteration will take the following form:

$$
\begin{aligned}
\hat{Q}^{(m+1)}= & \frac{r+\nu+2 C}{r+2 C[1-\cos (q)]+\gamma k^{2}} \hat{Q}^{(m)}(k, q) \\
& -\frac{\mathbb{F}\left[V_{n}(Y) Q_{n}^{(m)}(Y)\right]-\left(\theta^{(m)}\right)^{2} \mathbb{F}\left[\kappa\left|Q_{n}^{(m)}\right|^{2} Q_{n}^{(m)}\right]}{r+2 C[1-\cos (q)]+\gamma k^{2}},
\end{aligned}
$$

where $\theta^{(m)}$ is defined by the right-hand side of (17) with $Q$ set equal to $Q^{(m)}$. Typical examples of self-localized beams are shown in Figs. 2-4. In Fig. 2 we have a trap in both $n$ and $Y$ and the mode is localized equally in both directions. In Fig. 3, we depict a trap with $\alpha=1 / 2$ and $\beta=0.1$, which is much longer in the $Y$ direction than the discrete $n$. We also note that when the trap is only a function of $n(\beta=0)$, the corresponding mode is only localized in the $n$ direction; similarly it turns out that when the trap is localized in the $y$ direction (e.g., $\alpha=0$ ) then the mode is only localized in the $Y$ direction. Finally we find that when the trap is "turned off" $V_{n}=0$-then we find a new localized mode in the focusing nonlinear case when $\gamma \kappa=1$ (see Fig. 4).

In conclusion, a model describing wave propagation in optically modulated waveguide arrays is derived from Maxwell's equations. In the weakly guided regime, a discrete nonlinear Schrödinger equation with the addition of a bulk diffraction term and an external "optical trapping potential" is derived. In the defocusing regime, where in the absence of modulation no finite energy solitons are known, the induced optical trap prevents the beam from defocusing, resulting in a stable unstaggered localized mode. These results also establish a connection to the modeling of Bose-Einstein condensation where discrete optical lattices with a potential induced by a magnetic trap have been studied (cf. [15]).

M.J.A. was partially supported by the Air Force Office of Scientific Research under Grant No. F-49620-03-1-0250 and by the NSF under Grant No. DMS-0303756 M.I.W. was partially supported by the NSF under Grant No. DMS-0412305. K.J. acknowledges partial support from the University of Colorado.
[1] D. N. Christodoulides and R. J. Joseph, Opt. Lett. 13, 794 (1988).

[2] H. Eisenberg, Y. Silberberg, R. Morandotti, A. Boyd, and J. Aitchison, Phys. Rev. Lett. 81, 3383 (1998).

[3] N. Efremidis, S. Sears, D. N. Christodoulides, J. Fleischer, and M. Segev, Phys. Rev. E 66, 046602 (2002).

[4] J. Fleischer, M. Segev, N. Efremidis, and D. N. Christodoulides, Nature (London) 422, 147 (2003).

[5] R. W. Boyd, Nonlinear Optics, 2nd ed. (Academic Press, SanDiego, CA, 2003).

[6] M. J. Ablowitz and Z. H. Musslimani, Physica D 184, 276 (2003).

[7] A. Yariv, Optical Electronics in Modern Communications (Ox- ford University Press, Oxford, 1997).

[8] P. L. Kelley, Phys. Rev. Lett. 15, 1005 (1965).

[9] M. I. Weinstein and B. Yeary, Phys. Lett. A 222, 157 (1996).

[10] M. I. Weinstein, Nonlinearity 12, 673 (1999).

[11] B. Yeary, Ph.D. thesis, University of Michigan, 1996 (unpublished).

[12] H. A. Rose and M. I. Weinstein, Physica D 30, 207 (1988).

[13] R. H. Goodman, R. E. Slusher, and M. I. Weinstein, J. Opt. Soc. Am. B 19, 1635 (2002).

[14] M. J. Ablowitz and Z. H. Musslimani, Opt. Lett.(to be published).

[15] P. Meystre, Atom Optics (Springer, Berlin, 2001). 